\title{
Impact of In-Situ Radome Lightning Diverter Strips on Antenna Performance
}

\author{
Ana Vukovic, Phillip Sewell, Trevor M. Benson
}

\begin{abstract}
Lightning diverter strips are commonly used to protect the antenna and sensitive equipment within an airborne radome. This paper compares the impact of solid metallic and segmented diverter strips on the radiation properties of the enclosed antenna. Solid metallic and segmented diverter strips of different segment profiles, i.e., square, circular and diamond, are considered. The paper reports how the placement of diverters on the radome and their geometric detail affect the antenna parameters, namely reflection coefficient and far field pattern. Furthermore, the surface electric field intensity on segmented diverter strips is analyzed for different shapes, sizes and separations between the metallic segments.

Index Terms-Antennas, lightning, radomes
\end{abstract}

\section{INTRODUCTION}

$\mathrm{A}$ NTENNAS located within an aircraft nose and shielded by a dielectric radome are highly vulnerable to damage caused by lightning strike as this zone is likely to experience initial lightning attachments and first return strokes (Zone 1A) [1,2]. It is therefore critical to integrate lightning protection on the radome surface in order to intercept lightning strikes and divert them to the airframe and away from the antenna. Lighting protection on the radome typically uses two types of diverter strips namely, solid metallic diverter strips and segmented diverter strips. Solid metallic diverter strips are made of solid bars of metal that are glued to the outside of the frame and provide a metal path for lightning current [1-4]. On the other hand, segmented diverter strips consist of a series of conductive segments fastened to a resistive material that is glued to the surface of the radome. Segmented diverters conduct lightning current by creating an ionizing channel in small gaps between metallic segments under a strong external field. A variety of segmented diverter strips are in use with segments that differ in the shape, size and separation between them [3]. Most commonly used are diverters with circular, square or diamond segments [3]. Typically, dimensions of segmented diverter strips that are used on radomes have widths less than $1 \mathrm{~cm}$ and metallic segments whole size is of the order of $\mathrm{mm}$ with the usual spacing between segments of the order of $\mathrm{mm}$. An illustration of different types of segmented diverter strips is shown in Fig.1. A geometrical model of a radome with a base radius of $0.4 \mathrm{~m}$ and height of $0.45 \mathrm{~m}$ with eight $0.4 \mathrm{~m}$ long segmented diverters is shown in Fig.2a) with a zoomed in detail of a segmented diverter strip on the radome surface shown in

The Manuscript received November 5, 2019. The authors are with the George Green Institute for Electromagnetics research, School of Electrical and Electronic Engineering, The University of Nottingham, Nottingham University Park, NG7 2RD, Nottingham, UK. (email: ana.vukovic@nottingham.ac.uk)
Fig.2b). The typical recommended spacing between diverter strips is $30-45 \mathrm{~cm}[3,5]$.

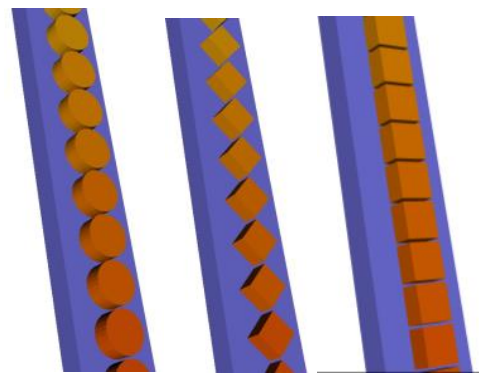

Fig.1. Geometry model of segmented diverters strips with various metallic segments.

An average aircraft is hit by a lightning once every 1000 hours. The design challenge is that the presence of diverter strips needs to have a low impact on the antenna performance and at the same time provide adequate protection from lightning strikes. These, in essence, requirements can be fulfilled once an understanding is reached on how the geometry of diverter strips and their placement on the radome surface affects both the enclosed antenna and the lightning current conduction. The geometry of diverter strips, their length, spacing and position needs to be optimized both in terms of lightning channel developments and at the same time ensure radar's operability [6].

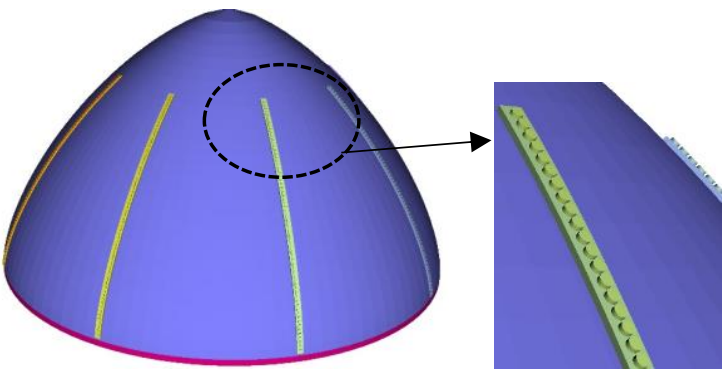

a)

b)

Fig.2. Geometrical detail of a) radome with integrated lightning protection and b) zoomed in detail of the segmented diverter strip on the radome surface.

Understanding the physical mechanisms of the lightning current conduction and the impact that segmented diverter geometry has on the breakdown voltage has mainly been

Digital Object Identifier XXX 
explored using experimental measurements of lightning channels on either flat or realistic radomes [3,4,7]. Full-wave simulation analyses have been applied to simpler 2D models of lightning channel ignition in segmented diverters [8,9] whilst simplified 3D simulation studies have been used to determine the streamer-leader development on radome diverter strips [6] and field distributions at the nose of the aircraft flying in thunderstorms [10]. These studies show that segmented diverters with larger metallic segments have lower breakdown voltages compared to those with smaller metallic segments but that smaller segments withstand higher current loads better [3]. The shape of the segments also affects the breakdown voltage, with diamond type segments having lower breakdown voltages than circular or square segments [9]. Furthermore, the effectiveness of the protection afforded using segmented diverters decreases significantly with increased length of the segmented strip, with some studies also showing that segmented diverter strips have limited strike capability compared to metallic diverters that have multi-strike capability [3]. In [10] the shielding effect of solid metallic and segmented strips on a spherical radome in the absence of an antenna was analyzed, and it was shown that solid diverter strips produce a shielding effect which increases with the number and the length of strips and that strong shielding causes undesirable field intensification at the front of the radome. The paper shows that segmented diverter strips do not cause a shielding effect which indicates that there will be no interference with the antenna radiation field until the breakdown along the strip is established [10].

On the other hand, the presence of lightning protection, either metallic or segmented diverter strips, on an otherwise electromagnetically transparent radome cover, will inevitably impact antenna performance. The analysis of the impact of diverter strips on antenna radiation has been dominated by experimental studies with the radome considered to be either cylindrical [4], spherical [10] and with a realistic profile $[4,11]$. Very few simulation studies have been reported and these consider simplified models. For example, in [4] the radome is considered to be completely transparent so that only the presence of the diverter strips was accounted for; it was shown that the presence of solid metallic diverters has a greater impact on antenna performance then segmented diverter strips. Experimental measurement on the impact of diverter strips on antenna are given in $[4,11]$ but the detail of the strips and/or radome not fully described and cannot be reproduced. However, both [4,11] agree with finding of [10] that solid diverter strips will interfere more with the antenna radiation pattern compared to segmented diverter strips.

The lack of a more comprehensive and detailed study of the effect that diverter strips have on antenna radiation patterns is due to two main difficulties. Firstly, in order to assess the impact of lightning protection on airborne antennas, broadband and fully coupled modelling of antenna-radome interactions on realistically sized geometries is required. This means that the antenna needs to be modelled in-situ which requires full-wave computational algorithms that are capable of handling the multiscale nature of the problem, specifically the large scale of the radome and, at the same time, the small geometrical features of the antenna. Our recent work on modelling installed antennas using a full-wave time-domain method shows that antenna performance is significantly changed when installed in realistic environments and is affected by both near and far field interactions [12,13].

Secondly, and most importantly, CAD models of a radome with diverter strips are not readily available. The individual components i.e. the radome and diverter strip are both defined in the Cartesian framework and it is impossible to generate a physically consistent geometry using Constructive Solid Geometry (CSG) techniques without creating unphysical artefacts at the radome-diverter interface which are detrimental for the purposes of accurate electromagnetic (EM) analysis. However, the CSG approach can be replaced by the Mean Value Coordinate (MVC) method that enables morphing of diverter strips onto the radome surface [14-17]. A step-by-step guide to the morphing of radome strips onto the radome surface is reported in [18] and will not be repeated in this paper. An example of the radome geometry with diverter strips obtained using MVC method is shown in Fig. 2.

The aim of this paper is to conduct a detailed investigation, using a full-wave 3D simulation tool, to assess the impact of diverter strips on in-situ antenna performance. This will be done by considering different lengths and separations between diverters, different types of diverters (i.e. metallic or segmented) and the geometry detail of segmented diverter strips i.e., the shape of conducting elements (square, circular or diamond). Furthermore, the surface electric field distributions for different types of segmented diverter strip geometries are examined to assess their performance under the conditions of breakdown and lightning current conductions.

For this purpose, we use a time-domain numerical method based on tetrahedral mesh we refer to as the Unstructured Transmission Line (UTLM) method [19]. The main benefit of this method lies in the fact that it uses a tetrahedral mesh which requires fewer sample points to capture curved and multiscale geometries that are dominant in this scenario. Unstructured meshes are routinely used with Finite Element (FE) methods [20] and have been developed for the Finite Difference Time Domain (FDTD) [21-23]. However, the TLM framework offers distinctly valuable features: a time-stepping algorithm that is obtained without approximations such as mass lumping; the electric and magnetic field samples are co-located in time and space and most importantly, the stability of a UTLM algorithm is provable a-priori on a cell-by-cell basis without resorting to estimators such as the Courant condition. For large scale simulations this is a critical advantage as late time instability has never been observed with the TLM. In the UTLM method the tetrahedral mesh is seamlessly combined with a Cartesian mesh which is used to model large empty space regions without unduly compromising the computational efficiency or with the complexity of bespoke sub-gridding techniques [24]. Being a time-domain method, the UTLM easily permits modeling of both linear, dispersive and nonlinear materials. Recent work has accounted for both electric and magnetic material losses [25], and the presence of carbon fiber panels as embedded thin film layers between the mesh cells [26]. Finally, an important advancement in the development of the UTLM method is its ability to deal with multiscale features of practical problems by deploying complexity reduction techniques in which small computational cells are coalesced into larger entities to reduce both pre-processing time and run time [27]. The UTLM method 
is not discussed in detail in this paper and supporting evidence for the accuracy and multiscale capability of the method, as well as its industrial deployment can be found in [19, 24-27].

The rest of the paper is organized as follows: Section II analyses the impact of solid metallic and segmented diverter strip parameters on antenna properties, Section III analyses the impact of the geometry of segmented diverter strips on the electric field distribution and Section IV summarizes the main conclusions of the paper.

\section{IMPACT OF IN-SITU RADOME DIVERTER STRIPS ON ANTENNA PERFORMANCE}

In this section the electromagnetic performance of the antenna inside a radome with lightning protection is analysed. The lightning protection is assumed to consist of a number of diverter strips that are either all metallic or segmented diverter strips. Typical design parameters such as the length of diverters, the separation between diverter strips, and the shape of diverter segments i.e. circular diamond or square, are considered.

The radome is considered to have an ogive profile described by the equation $x^{2}+y^{2}=(2 R / L)^{2}\left(L^{p}-z^{p}\right)^{2 / p}$, where the $\mathrm{z}$ coordinate is defined along the axis of the radome, $L$ indicates the length of the radome, $R$ is the base radius and the parameter $p=1.449$ defines the radome profile. The radome base radius and length are fixed to be $R=0.4 \mathrm{~m}$ and $L=0.45 \mathrm{~m}$ respectively. In all cases the half-wave monolithic radome is made of a glass composite of thickness $24.4 \mathrm{~mm}$ and relative dielectric constant $\varepsilon_{\mathrm{r}}=4.2$ which is designed to operate at $3 \mathrm{GHz}$. The radome base is circular with a thickness of $5 \mathrm{~mm}$. Radome material losses are neglected. A Vivaldi antenna operating at $3 \mathrm{GHz}$ is placed inside the radome. The antenna is printed on a dielectric substrate with $\varepsilon_{\mathrm{r}}=3$ and is based upon [28]. The width, height and thickness of the substrate are $40 \mathrm{~mm}, 55 \mathrm{~mm}$ and $1.5 \mathrm{~mm}$, respectively. The antenna is fed by a coaxial line whose inner radius is $0.375 \mathrm{~mm}$ and outer radius is $0.875 \mathrm{~mm}$. The dielectric constant of coaxial insulator is $\varepsilon_{\mathrm{r}}=2.25$. The radome with in-situ antenna as per given dimensions is shown in Fig.3. Fig.3. also shows the H-plane $(\theta)$ and E-plane $(\phi)$ of the antenna pattern.

Both types of diverter strips are considered namely metallic and segmented diverter strips. The following diverter parameters are kept constant throughout the paper:

- the width of the solid metallic and segmented diverter strips is $1 \mathrm{~cm}$;

- the thickness of the solid metallic layer or metallic segments is taken to be $2 \mathrm{~mm}$;

- the metallic layer and metallic segments are placed on an insulating layer of $3 \mathrm{~mm}$ thickness and dielectric constant $\varepsilon_{\mathrm{r}}=2$.

As an illustration the geometries of the segmented diverter strips with circular, diamond and square segments are shown in Fig.1) with separation between the segments of $0.5 \mathrm{~mm}$. The diameter of circular segments is $5 \mathrm{~mm}$ and the diamond and square segments are of the same square shape but oriented differently with the side length of $3.54 \mathrm{~mm}$. The length of the diverter is assumed to be that of a straight strip.

The whole problem is meshed with a hybrid mesh that is a combination of a $5 \mathrm{~mm}$ cubic mesh and a tetrahedral mesh. The antenna near field is meshed more finely with $1 \mathrm{~mm}$ hybrid mesh as described in [13]. The meshed radome with lightning protection is shown in Fig.4a) and the meshed detail of the diverter strip is shown in Fig.4b) demonstrating the hybrid cubic-tetrahedral and multi-scale nature of the deployed mesh.

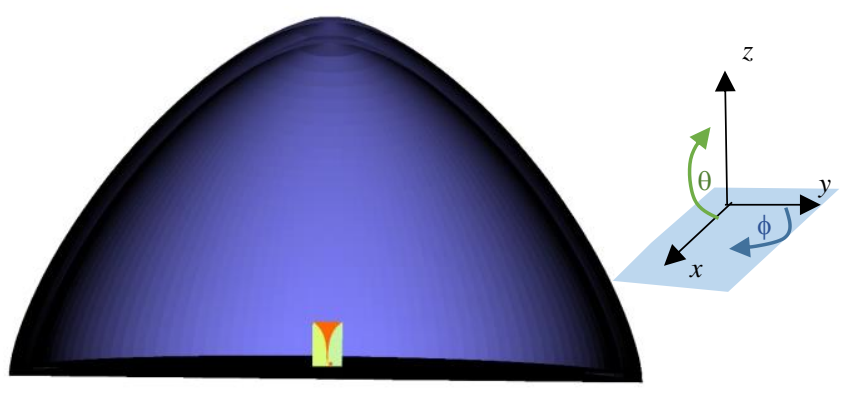

Fig.3. Geometry model of the antenna inside the radome with no lightning protection and b) H-pane $(\theta)$ and E-plane $(\phi)$ definition.

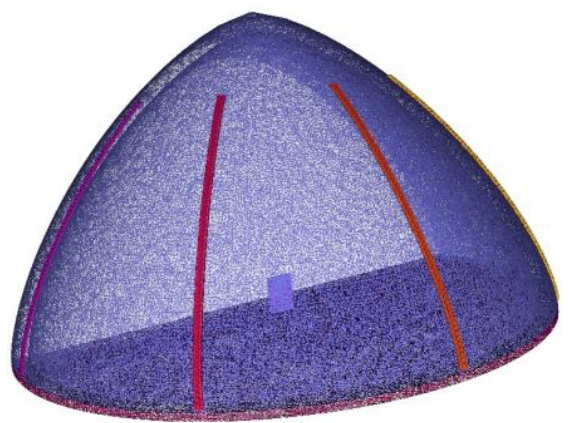

a)

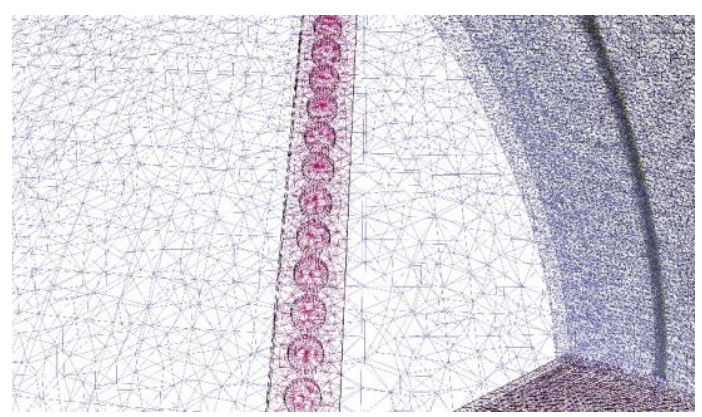

b)

Fig.4. a) Meshed geometry of the radome with lightning protection and insitu antenna, and b) mesh detail around the segmented diverter strip.

The antenna is excited with the fundamental TEM mode of the coaxial feed modulated by a time domain pulse with $3 \mathrm{~dB}$ frequencies of $1.8 \mathrm{GHz}$ and $4.6 \mathrm{GHz}$. The fundamental TEM mode is obtained as an eigen-solution of the discretized twodimensional cross section of the coaxial cable [13, 29]. All simulations in this paper are run on 60 processor cores of a commodity cluster for 2 million time steps. The threshold for forming cell clusters is $5 \mu \mathrm{m}$ and the timestep is $0.018 \mathrm{ps}$ [19]. As an example, the radome problem with $0.4 \mathrm{~m}$ long metallic diverter strips had 981057 tetrahedra, 310243 cuboids, 181581 clusters with the largest coalesced cluster having 63 cells. The overall simulation runtime was 7 hours. In contrast, the radome 
with segmented diverters of $0.4 \mathrm{~m}$ length had 1.2 million tetrahedra, 310551 cuboids, 189544 clusters with the largest coalesced cluster having 8 cells, and overall simulation runtime of 9.5 hours.

Fig.5 shows how the reflection coefficient of the antenna, $\mathrm{S}_{11}$, is affected by the presence of radome lightning protection by comparing it with the reflection coefficient of the antenna inside the radome with no lightning protection. The segmented diverter strips with circular segments of $5 \mathrm{~mm}$ diameter and 1 $\mathrm{mm}$ spacing between segments are considered. In order to fit in with the recommended spacing between diverters of 30-45 cm [3] the radome is fitted with a total of eight diverter strips separated by $31 \mathrm{~cm}$ at the radome base. Fig.5 also compares the impact of diverter strips of different lengths, namely $0.15 \mathrm{~m}, 0.3$ $\mathrm{m}$ and $0.4 \mathrm{~m}$.

Fig.5a) compares the $S_{11}$ of the antenna within a radome with and without solid metallic diverters of different lengths and Fig.5b) compares the antenna's $S_{11}$ with and without segmented diverter strips of different lengths. Comparing Fig.5a) and Fig.5b) it can be seen that in both the case of metallic or segmented diverter strips the presence of diverters of various lengths does not significantly affect the reflection coefficient of the antenna. The only impact is the increase in reflection at the resonant frequency of $3 \mathrm{GHz}$ by $6 \mathrm{~dB}$ for metallic diverters and by $5 \mathrm{~dB}$ for segmented diverters and, in both cases, a slight shifting of the resonant frequency to higher frequencies.

Fig.5a) compares the $S_{11}$ of the antenna within a radome with and without solid metallic diverters of different lengths and Fig.5b) compares the antenna's $S_{11}$ with and without segmented diverter strips of different lengths. Comparing Fig.5a) and Fig.5b) it can be seen that in both the case of metallic or segmented diverter strips the presence of diverters of various lengths does not significantly affect the reflection coefficient of the antenna. The only impact is the increase in reflection at the resonant frequency of $3 \mathrm{GHz}$ by $6 \mathrm{~dB}$ for metallic diverters and by $5 \mathrm{~dB}$ for segmented diverters and, in both cases, a slight shifting of the resonant frequency to higher frequencies.

Fig.6 compares the impact of the separation of the metallic segments in segmented diverters on the antenna $S_{11}$ parameter. As in Fig.5, eight segmented diverters of length $0.4 \mathrm{~m}$ are considered. Circular metallic segments of $5 \mathrm{~mm}$ diameter are considered with spacing between segments taken to be $1 \mathrm{~mm}$, $0.5 \mathrm{~mm}$ and $0.3 \mathrm{~mm}$. Fig. 6 shows that reducing the separation between the metallic segments can affect the $S_{11}$ of antenna but has a higher impact at higher frequencies.

Fig.7 compares the impact on the diverter strips on the threedimensional (3D) radiation pattern. Antenna patterns are shown for the case of antenna enclosed in a radome with no lightning protection, Fig.7a), in a radome with 8 solid metallic diverters, Fig.7b), and in a radome with segmented diverter with circular segments of $5 \mathrm{~mm}$ diameter and separation between segments of $1 \mathrm{~mm}$, Fig.7c). All diverters are assumed to be $0.4 \mathrm{~m}$ long.Fig.7 shows that the metallic diverters have the highest impact on the radiation pattern whilst this particular type of segmented diverters has relatively small impact on antenna radiation pattern. Comparing these results with that of [4] it can be seen that, although the antennas and radome are different, the impact of lightning protection on antenna radiation pattern is similar.
In order to clearly identify the impact of the diverter on the radiation pattern the $\mathrm{H}$-field and $\mathrm{E}$-field radiation patterns will now be analyzed in more detail. A total of eight solid metallic or segmented diverters are placed on the radome and have length of $0.4 \mathrm{~m}$. Segmented diverter strips have circular segments of $5 \mathrm{~mm}$ diameter and $1 \mathrm{~mm}$ separation between segments, as in Fig.5. Fig.8a) compares the E-plane radiation pattern and Fig. 8 b) compares the H-plane radiation pattern for antenna enclosed in a radome with no lightning protection, in a radome with metallic diverters and in a radome with segmented diverters. Fig. 8 shows that metallic strips have a greater impact on the far field pattern than the segmented diverters strips. Furthermore, comparing Fig.8a) and Fig.8b) it can be seen that the far field radiation in the E-plane is more affected by the presence of lightning protection.

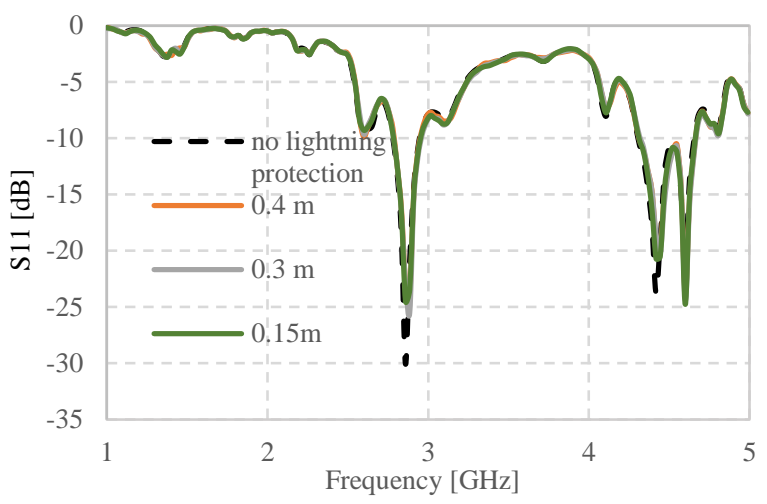

a)

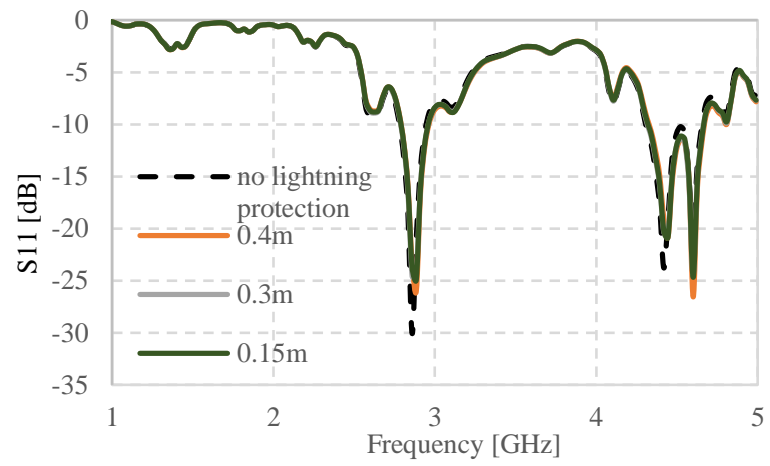

b)

Fig.5 Comparison of the $S_{11}$ parameter of an antenna placed inside a radome with and without a) solid metallic strips and b) segmented diverters strips of various lengths. Diverter strips have lengths of $0.15 \mathrm{~m}, 0.3 \mathrm{~m}$ and $0.4 \mathrm{~m}$.

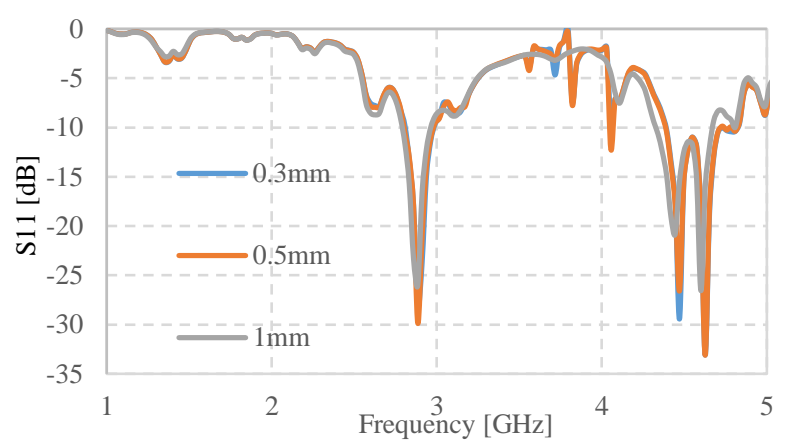

Fig.6 Comparison of the $S_{11}$ parameter of an antenna placed inside a radome with 8 segmented diverters strips of $0.4 \mathrm{~m}$ length. The segments are assumed to be circular of $5 \mathrm{~mm}$ diameter and with separation between segments of $0.3 \mathrm{~mm}$, $0.5 \mathrm{~mm}$ and $1 \mathrm{~mm}$. 


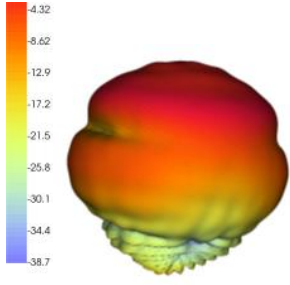

a)

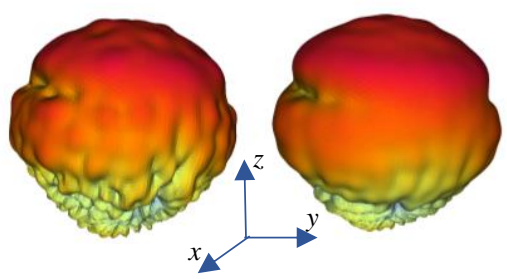

b)

c)
Fig.7. 3D radiation pattern of the Vivaldi antenna enclosed in a radome with a) no lightning protection, b) with 8 solid metallic diverters and c) with 8 segmented diverters. Color scale is in $\mathrm{dB}$.

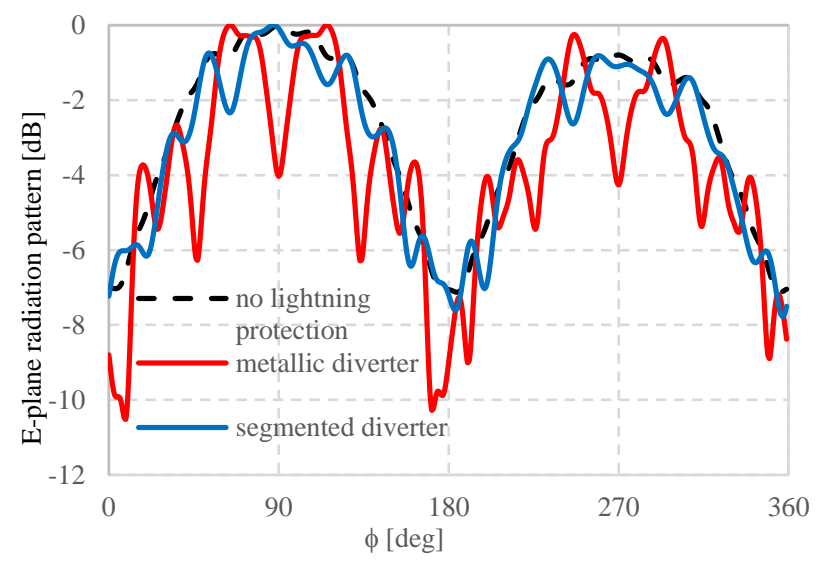

a)

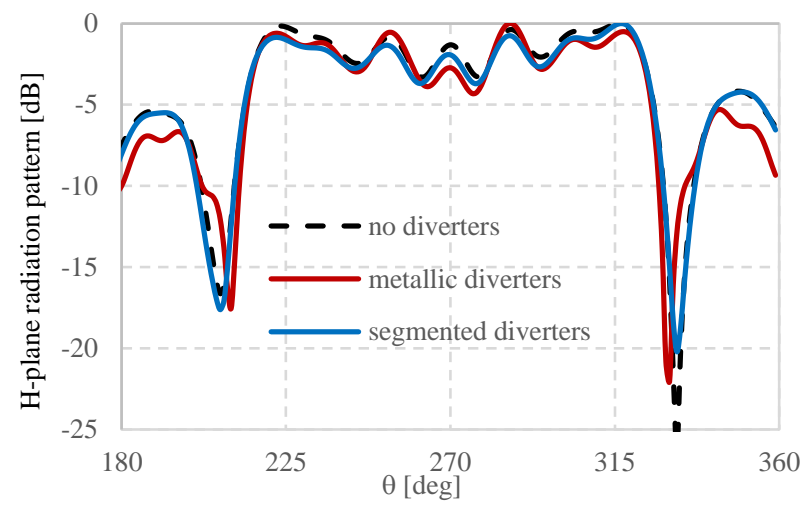

b)

Fig.8 Comparison of the far field in a) the E-plane and b) the H-plane of an antenna placed inside a radome with no lightning protection (dashed black line), within a radome with eight solid metallic diverter strips (red) and within a radome with eight segmented diverters (blue). All diverters are $0.4 \mathrm{~m}$ long. Segmented diverters have metallic segments of $5 \mathrm{~mm}$ diameter separated by 1 $\mathrm{mm}$.

As the presence of solid metallic diverters impacts the far field pattern more, Fig.9 explores how the length of the solid metallic diverter strips affects the radiation pattern. Fig.9 compares the radiation pattern of the antenna within a radome with no lightning protection (black dashed line), with the radome with eight diverter strips of lengths $0.15 \mathrm{~m}$ (green), 0.3 $\mathrm{m}$ (red) and $0.4 \mathrm{~m}$ (blue). Comparing Fig.9a) and Fig.9b) it can be seen that as the length of the strips is increased the ripples in the E-plane far field pattern are also increasing.

Fig.10a,b) explores how the separation between diverter strips affects the far field radiation pattern. Fig.10a) compares the E-plane far field radiation pattern of antenna in a radome with no lightning protection (black dashed line) with an antenna enclosed with a radome with lightning protection consisting of 4 solid metallic strips (green line), 8 solid metallic strips (blue line) and 12 solid metallic strips (red line). Fig.10b) shows the same information but for segmented diverter strips. In all cases the length of the strip is assumed to be $0.4 \mathrm{~m}$. Fig. 10 shows that increasing the number of metallic strips, i.e. decreasing the separation between them, can significantly affect the radiation pattern in both planes. The recommended separation is 30-45 $\mathrm{cm} \mathrm{[3]} \mathrm{and} \mathrm{it} \mathrm{can} \mathrm{be} \mathrm{seen} \mathrm{that} \mathrm{in} \mathrm{the} \mathrm{case} \mathrm{of} 12$ strips where the separation at the base of the radome becomes $0.21 \mathrm{~m}$, the overall radiation pattern is significantly affected by the presence of solid metallic strips.

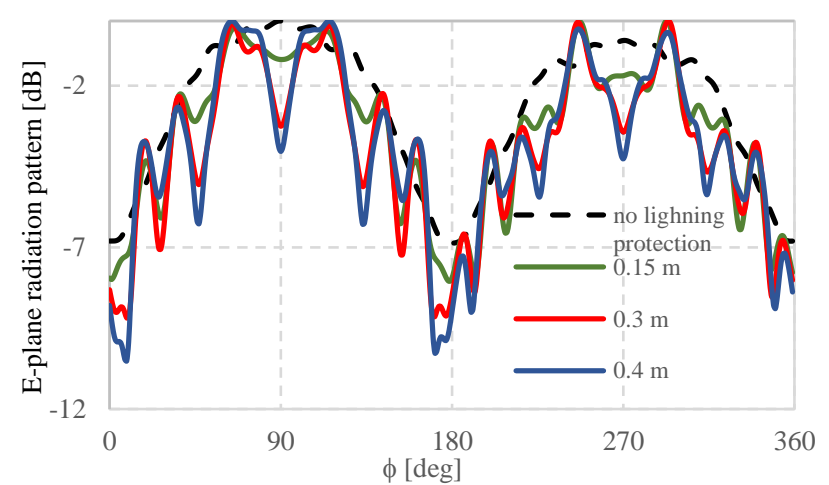

a)

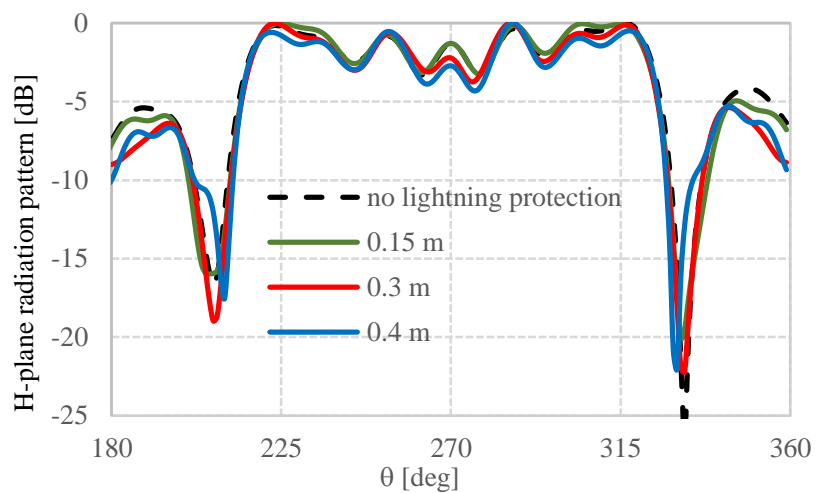

b)

Fig.9 Comparison of the far field in a) the E-plane and b) the H-plane of an antenna placed inside a radome with no lightning protection (dashed black line), with antenna within a radome with eight solid metallic diverter strips of lengths $0.15 \mathrm{~m}$ (green line), $0.3 \mathrm{~m}$ and (red line) and $0.4 \mathrm{~m}$ (blue line).

Fig.11a,b) shows the same information but for segmented diverter strips with circular segments. The geometry of the segmented strips is the same as in Fig.5 i.e. metallic segments are of $5 \mathrm{~mm}$ diameter separated by $1 \mathrm{~mm}$. Diverter strips are 0.4 $\mathrm{m}$ long. Fig.11 shows that similarly to the Fig.10, the E-plane is more affected by the presence of segmented diverters but the impact is still much smaller when compared to the equivalent case of metallic diverters in Fig.10. This result indicates that reducing separation between diverters is less critical in the case of segmented diverter strips than in the case of solid metallic strips. 


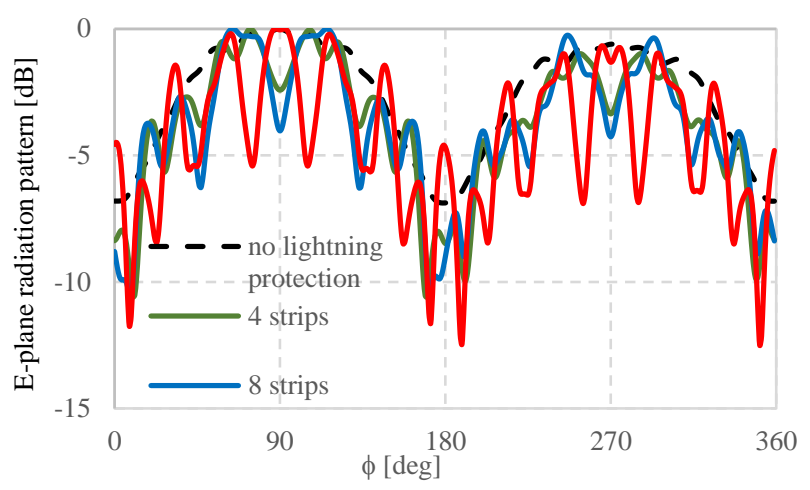

a)

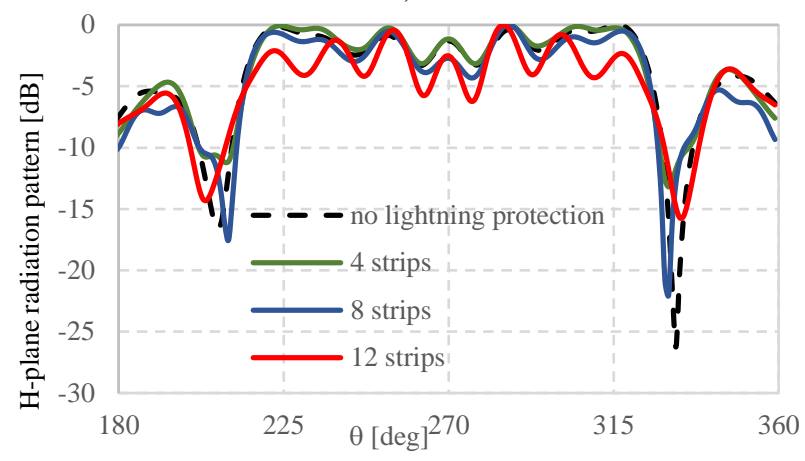

b)

Fig.10 Comparison of the far field in a) the E-plane and b) the H-plane of an antenna placed inside a radome with no lightning protection (dashed black line) with antenna within a radome with 4 metallic strips (green line), 8 metallic strips (blue line) and 12 metallic strips (red line).

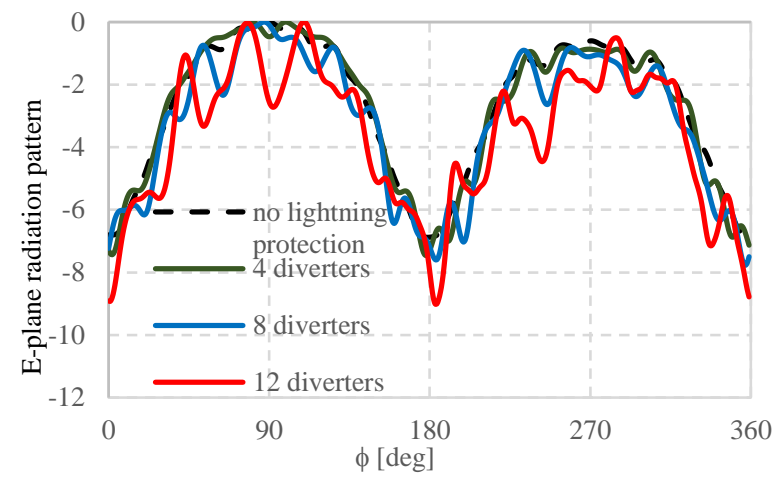

a)

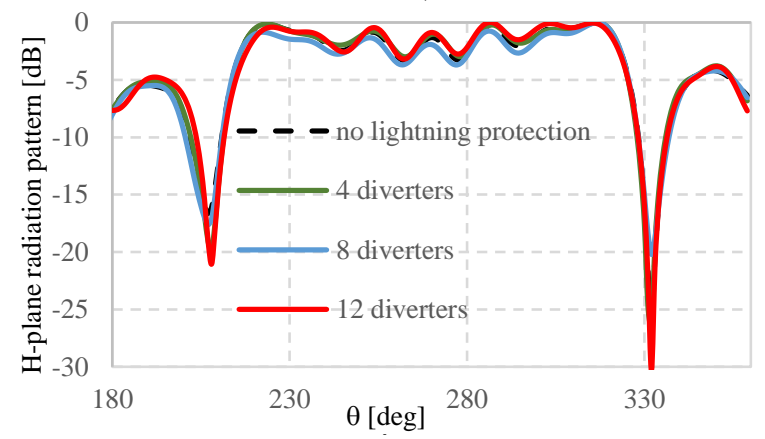

b)

Fig.11 Comparison of the far field patterns in a) the E-plane and b) the $\mathrm{H}$ plane of an antenna placed inside a radome with no lightning protection (dashed black line), with antenna within a radome with 4 diverter strips (green line), 8 diverter strips (blue line) and 12 diverter strips (red line).
Fig.12 investigates how the separation between metallic segments affects the far field radiation pattern of the radome antenna. The radome has eight segmented diverters with circular metallic segments of $5 \mathrm{~mm}$ diameter and strip length of $0.4 \mathrm{~m}$. Three cases are considered, namely segmented diverters with separation between metallic segments of $1 \mathrm{~mm}$ (green), 0.5 $\mathrm{mm}$ (blue) and $0.3 \mathrm{~mm}$ (red). Fig. 12 shows that decreasing the separation between the segments results in increased disturbance of the antenna field pattern, specifically increasing the ripple in the E-plane and reducing the intensity in the main lobe in the H-plane. It can be argued that decreasing the segment separation is making the segmented diverter strip appear more like a metallic strip and for this purpose Fig.13 compares the effect of metallic diverter strips with segmented diverter strips with segment separation of $0.3 \mathrm{~mm}$. A radome with 8 diverters of $0.4 \mathrm{~m}$ length is considered in both cases. Fig. 13 shows that the impact of these two types of diverters on the antenna far field characteristic is very similar. In practice segmented diverter strips have separations below $0.5 \mathrm{~mm}$ for good lightning protection [9] and this shows that the impact of segmented diverter strips on antenna radiation is not insignificant as stated in $[4,10]$, and will depend upon the geometry detail of diverter strips.

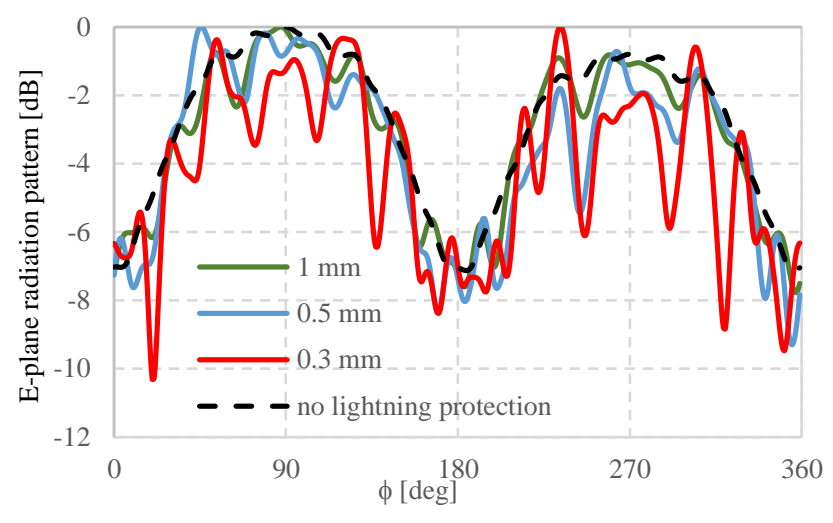

a)

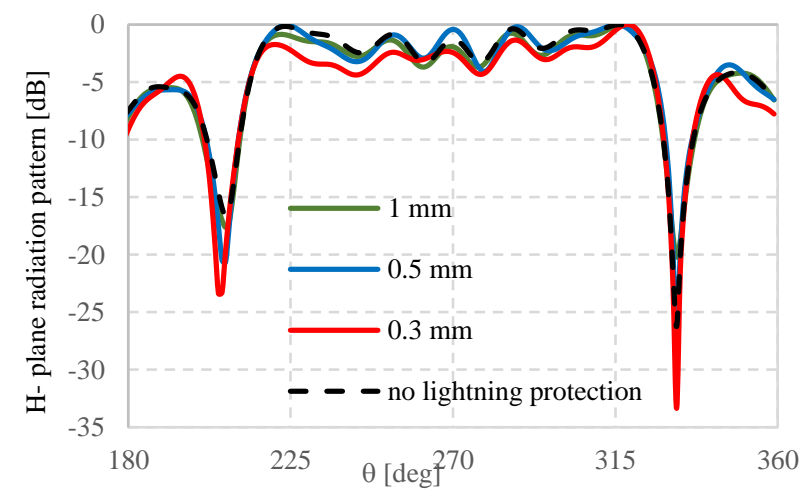

b)

Fig.12. Comparison of the far field patterns in a) the E-plane and b) the $\mathrm{H}$ plane of an antenna inside a radome with eight segmented diverters strips of varying separation between metallic segments: no diverter (black dashed line), $1 \mathrm{~mm}$ (green line), $0.5 \mathrm{~mm}$ (blue line) and $0.3 \mathrm{~mm}$ (red line). Radome with no lightning protection is given as a reference.

Having established how the separation of metallic segments in diverters affects antenna performance, we now consider the 
effect that the shape of the diverter segments has on antenna radiation and near field. Fig.14 compares the far field of an antenna enclosed in a radome with no lightning protection (black dashed line), with the same antenna enclosed in a radome with segmented diverter strips having circular (red), square (blue) and diamond (green) shape. In all cases the separation between the segments is taken to be $0.3 \mathrm{~mm}$, the length of the diverters is $0.4 \mathrm{~m}$ and the radome has 8 segmented diverter strips. Fig. 14 shows that segmented diverter strips with circular and square metallic segments interfere more with antenna compared to diverters with diamond segments.

Fig. 15 assesses how different types of segmented diverter strips affect the antenna reflection coefficient by comparison against the reflection coefficient of an antenna in-situ radome with no lightning protection. All segmented diverters have 0.3 $\mathrm{mm}$ separation between metallic segments. Fig. 15 shows that the major impact of segmented diverter strips is at higher frequencies and that their presence causes slight shift of the resonant frequency of the antenna. Fig. 15 shows that diverter strips with square segments have the biggest impact on the antenna reflection coefficient especially at higher frequencies. Fig.14 and Fig.15 both confirm that diverters with diamond segments would be a preferred choice for diverters as they interact least with antenna radiation pattern and the reflection coefficient.

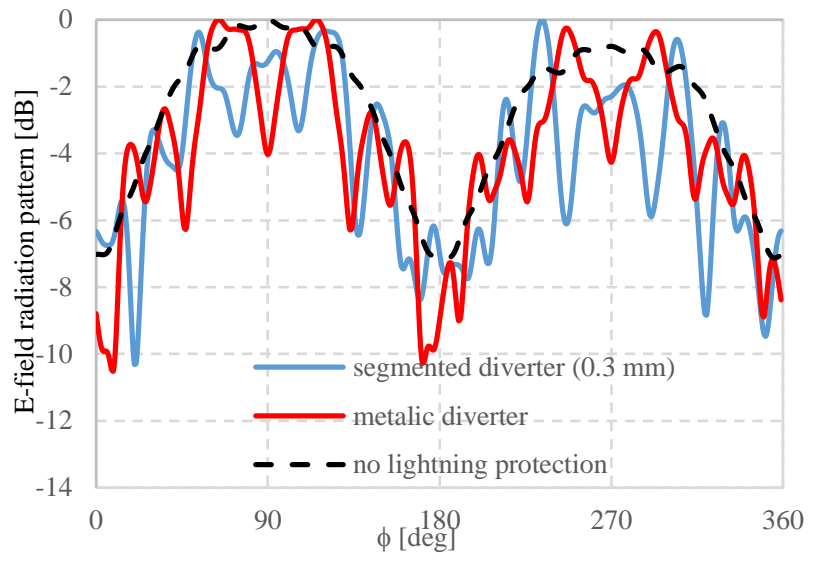

a)

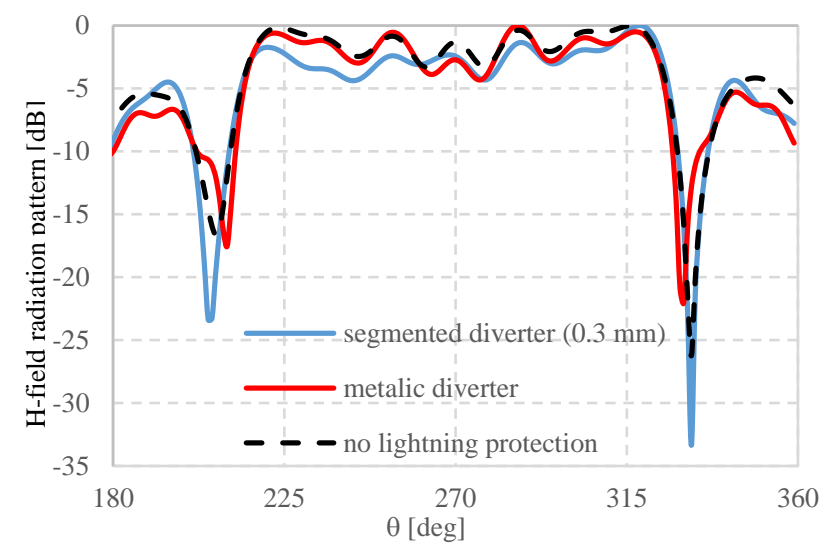

b)

Fig.13 Comparison of the antenna far field in a) the E-plane and b) the Hplane of an antenna inside the radome with no lightning protection (black dashed line), eight metallic diverters (red line) and eight segmented diverter strips with $0.3 \mathrm{~mm}$ separation between metallic segments (blue line). Radome with no lightning protection is given as a reference.

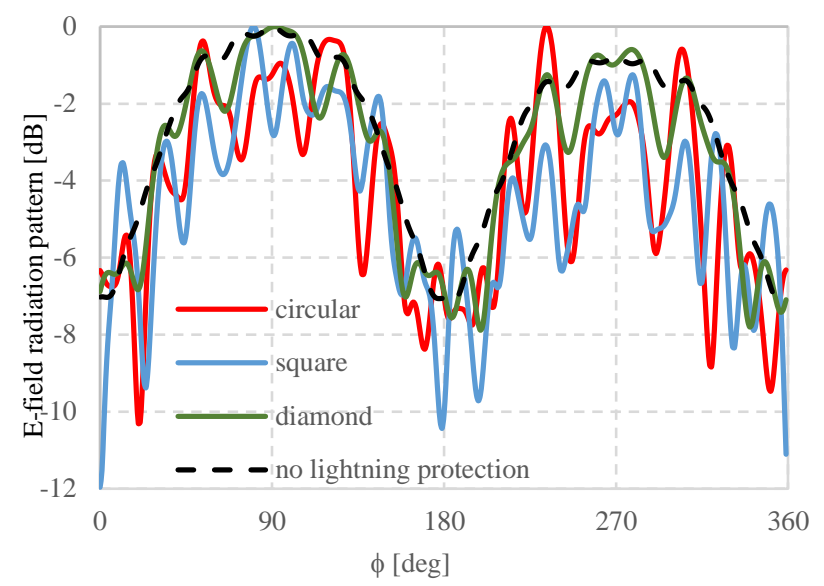

a)

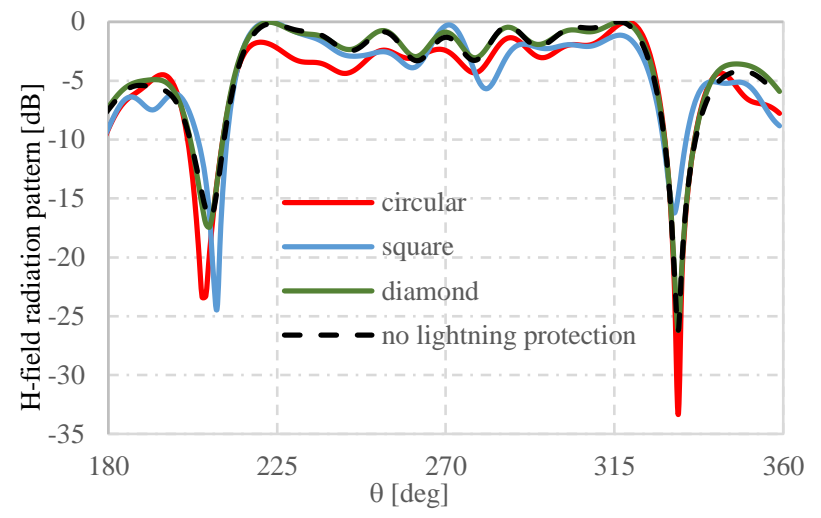

b)

Fig.14 Comparison of the antenna far field in a) the E-plane and b) the $\mathrm{H}$ plane of an antenna inside a radome with no lightning protection (black dashed line) with that of antenna enclosed in a radome with segmented diverter strips with circular (red), square (blue) and diamond (green) shaped elements.

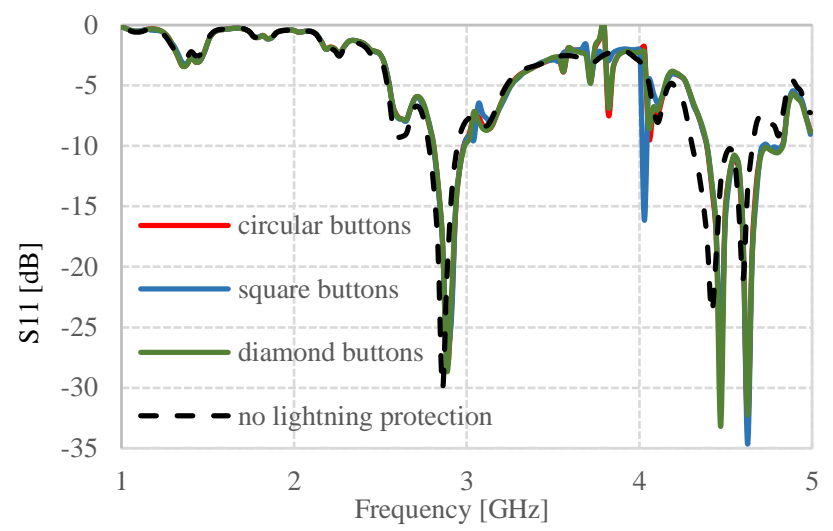

Fig. 15 Comparison of the reflection coefficient of an antenna placed inside a radome with no lightning protection (black dashed line) with that of antenna enclosed in a radome with eight segmented diverter strips with circular (red), square (blue) and diamond (green) shaped elements.

\section{IMPACT OF THE DIVERTER GEOMETRY ON THE SURFACE ELECTRIC FIELD INTENSITY}

The performance of the diverter strips in the presence of the strong incoming field is investigated next. Examining the field intensity between segments permits an assessment of the sensitivity to breakdown. The structure analyzed consists of a segment of a diverter strip on which appropriate boundary 
conditions (BC) are applied. The schematic of the structure is given in Fig.16a) with open BCs specified at minimum and maximum positions against the $x$-direction and at the symmetry plane $(y=0)$ and matching boundary conditions applied on other boundaries defined to be $10 \mathrm{~mm}$ away from the structure. The front and side view of the full problem meshed with a combination of tetrahedral and a global cubic $0.5 \mathrm{~mm}$ mesh with eight times finer mesh around the diverter structure is shown in Fig.16b). A zoomed in detail of the fine mesh around the segments is shown in Fig.16c).

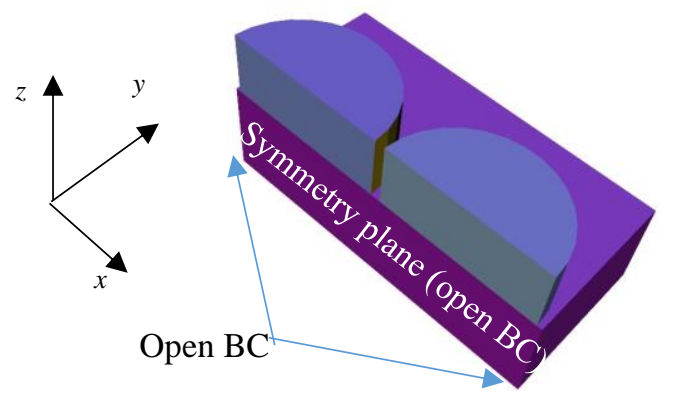

a)

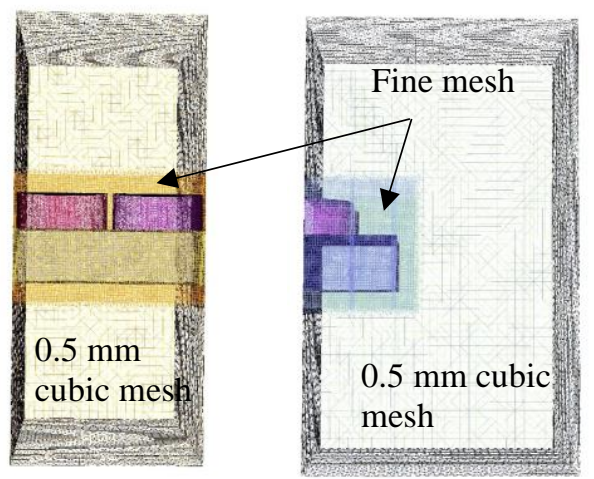

b)

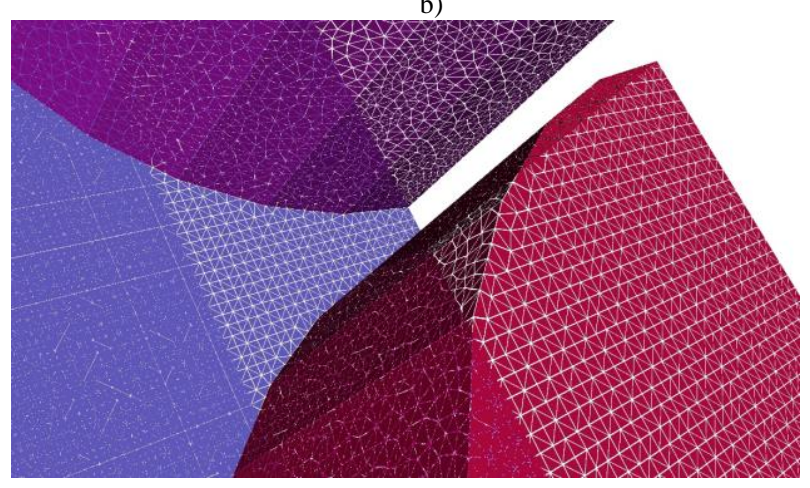

c)

Fig.16 a) Computation model of a two element segmented diverter with circular segments and with a symmetry plane; b) meshed problem showing global cubic $0.5 \mathrm{~mm}$ mesh and fine mesh around the metallic segments; c) zoomin detail of the meshed problem. Colored by geometry detail.

All segmented diverters considered had a substrate thickness of $2 \mathrm{~mm}$, metal segment thickens of $2 \mathrm{~mm}$, and segment diameter of $5 \mathrm{~mm}$. The separation between the segments was taken to be $0.3 \mathrm{~mm}$. A diverter strip was subjected to a strong $x$-polarized Gaussian signal with a spectrum from $1 \mathrm{kHz}$ to 300 $\mathrm{MHz}$ that propagates in the negative $z$-direction. Fig.17 compares the surface electric field in the diverter segment for the circular, diamond and square shaped metallic segments. It can be seen that the maximum surface electric field in the case of a diamond segment is twice that for the case of the circular segment and larger than for the case of square segments indicating that the diamond segment shape will have an earlier breakdown. This is in agreement with the 2D analysis of [9] where diamond shaped elements had lower breakdown voltages compared to the square and circular shaped segments.

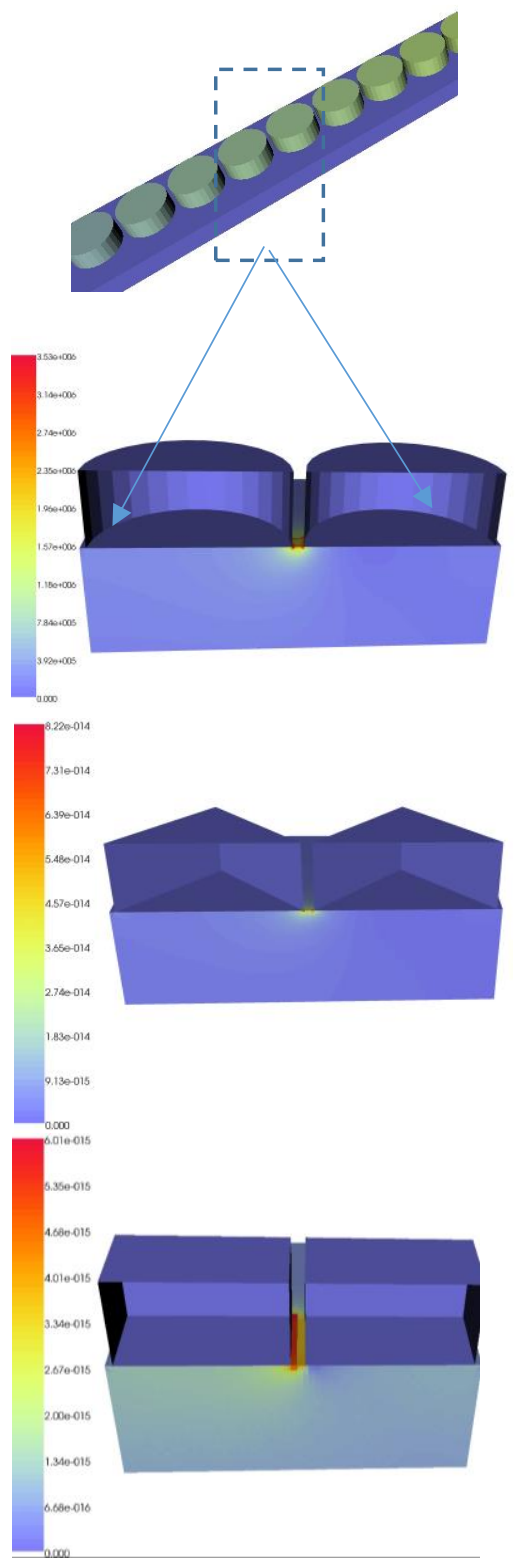

Fig.17 Comparison of surface electric fields in segmented diverters between a) circular, b) diamond and c) square metallic segments. Colored by the intensity of the surface electric field.

Fig. 18 compares the time waveform of the average $x$-directed surface electric field across the gap between the segments for each of the three diverter geometries. Fig.18 shows that the diamond segments produce the largest peak surface field compared to square and circular elements. 


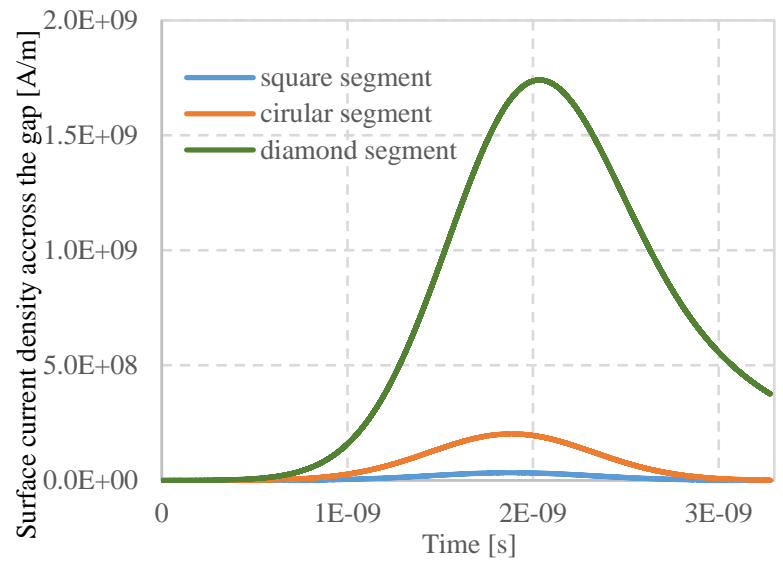

Fig.18 Comparison of the time waveform of the surface electric field across the gap between diamond (green), circular (red) and square (blue) segment diverter strips.

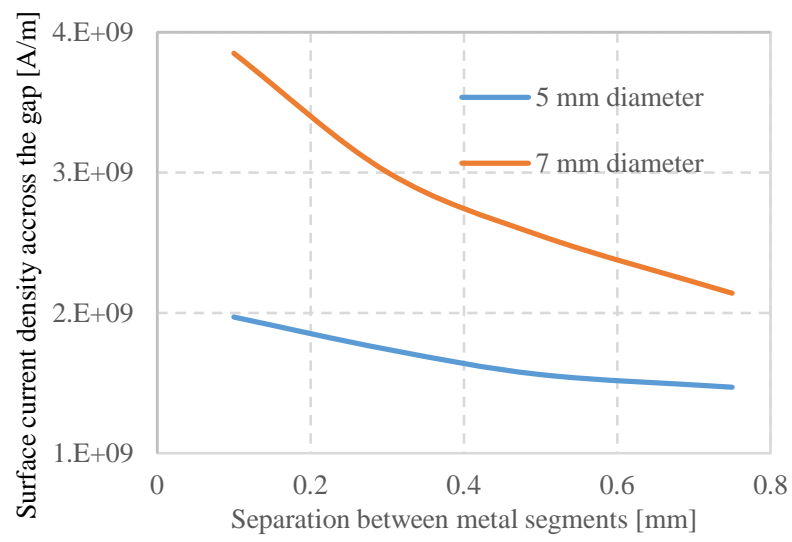

Fig.19 Comparison of the surface current density for two different sizes of metallic segments $(5 \mathrm{~mm}$ and $7 \mathrm{~mm}$ in diameter) and for different separations between the segments.

Fig. 19 explores how the size and the separation between the metallic segments affects the surface electric field across the gap between the metallic segments. Diamond shaped segments are considered of $5 \mathrm{~mm}$ and $7 \mathrm{~mm}$ diameter. It can be seen that as the size of the metallic segments is increased the surface electric field also increases. This suggests that larger segments are better as they will produce lower breakdown voltages; however, larger segments will also be more "visible" to enclosed antenna so this need to be balanced carefully. Fig.19 shows that reducing the separation between segments also increases the surface current density.

\section{CONCLUSION}

The paper has assessed how radome lightning protection in the form of solid metallic or segmented diverter strips affects the operation of the enclosed antenna. Different lengths and separation between diverters as well as the fine detail of the geometry of the segmented diverter strips namely the shape and separation between the segments were considered. The results show several important conclusions. In terms of diverter placement on radome, the results show that for both the solid metallic and segmented diverter strips increasing the length and separation between diverters generally increases their impact on the antenna, particularly on the far field pattern. The separation between metallic segments in segmented diverters is crucial in that smaller separations increase their impact on antenna far field. Whilst the paper shows that segmented diverter strips with separation between segments of $0.5 \mathrm{~mm}$ and greater have smaller effect on antenna than an equivalent metallic diverter strip, for practical values of separations of $0.3 \mathrm{~mm}$ the segmented diverter strip has comparable effect on antenna far field as the equivalent metallic diverter strip. Furthermore, the shape of the metallic segments in segmented diverters also affects how the antenna "sees" the radome lightning protection. The results show that diamond shaped segments have the smallest impact on the antenna compared to diverters with circular and square segments even with practical separations of $0.3 \mathrm{~mm}$. This paper thus disagrees with conclusions of $[4,10]$ that segmented diverter strips do not produce any visible shielding effects and concludes that the amount of shielding will strongly depend upon the actual geometry detail of the segmented strips. This paper further analyzes the performance of the segmented diverter strips in the presence of the strong electrical field by analyzing surface current density on the strip. The results show that surface electric field between metallic segments is strongest for the case of diamond shaped segmented diverters and weakest for the diverters with square metallic segments. The surface electric field between metallic segments increases with larger size of the metallic segments and with reduced separation between them. This new insight reinforces the importance of 3D multi-scale modelling in airborne radome design.

\section{ACKNOWLEDGMENT}

The authors wish to thank Dr S. Earl and Prof. C. Jones of BAE SYSTEMS for many valuable discussions.

\section{REFERENCES}

[1] J. A. Plumer, J. D. Robb, "The direct effects of lightning on Aircraft", IEEE Trans. On Elect. Compat., Vol.24, no.2, pp.158-172, 1982.

[2] J. A. Plumer, L. C. Hoots, "Ligthning protection with segmented diverters", IEEE Int. Sym. Electromagnetic Compatibility, pp.196-203, 1978.

[3] C. Karch, M. Calomfirescu, M. Rothenhäusler, C. Brand, and H. Meister "FFS: Lightning strike protection of radomes - an overview", Conf. Deutscher Luft- und Raumfahrtkongress, 2017.

[4] D. Yanchao, X. Xiu, H. Pingdao, "Research on aircraft radome lightning protection based on segmented diverter strips", Proc. of International symposium on Electromagnetic Compatibility, EMC Europe 2017, France, September 2017.

[5] https://patents.google.com/patent/US7120004B2/en

[6] C. Karch, W. Wulbrand, H. W. Zaglauer, C. J. Hardwick, "An approach to determining radome diverter strip geometry", Proc. Of International Conference on Lightning and Electricity (ICOLSE), Vol.2, Blackpool, UK, 2003.

[7] A. Ulmann, P. Brechet, A. Bondiou-Clergerie, A. Delannoy, P. Lalande, P. Blanchet, P. laroche, G. L. bacchiega, I. Gallimberti, "New investigations of the mechanism of lightning strikes to radomes, Part I: Experimental study in high voltage laboratory", ICOLSE, 2001, Avaliable: https://www.semanticscholar.org/paper/New-Investigationsof-the-Mechanisms-of-Lightning-I-UlmannBrechet/802ceade4463fcc93d5778595a643805d693beb0

[8] A. Elkalsh, A. Vukovic, T. M Benson, P. D. Sewell, "Coupled electro thermal model for lightning strike prediction and thermal modelling using the TLM Method", IEEE Journal on Multiscale and Multiphysics Computational Techniques, Vol.2, pp.38-48, March 2017.

[9] H. Chen, "Plasma discharge characteristics of segmented diverter stris subject to lightning strike," Plasma Sci. Technol., vol. 21, 025301, pp. 1-13, 2019. 
[10] N. I. Petrov, A. Haddad, H. Griffiths, R. T. Waters, "Lightning strikes to aircraft radome: Electric field shielding simulation", Proc. $17^{\text {th }}$ International Conference on Gas Discharges and their applications", Sept 2017.

[11] A. Piche, G-.P. Piau, C. Bernus, F. Campagna, D. Balitrand, "Prediction by simulation of electromagnetic impact of radome on typical aircraft" The 8th European Conference on Antennas and Propagation (EuCAP), pp.3205-3208, 2014.

[12] A. Vukovic, P. Sewell, X. Meng, T.M. Benson, "Installed antenna performance in airborne radomes of different profiles", ACES Express Journal, Vol.33, No.3, pp.343-346,March 2018.

[13] A. Vukovic, P. Sewell, T. M Benson, "Holistic appraisal of modelling installed antennas for aerospace applications", IEEE Trans on Antennas and Prop., Vol. 67, no.3, 1396-1490, March 2019.

[14] B. Lévy, S. Petitjean, N. Ray, J. Maillot: "Least Squares Conformal Maps for automatic texture atlas generation" ACM Transactions on Graphics, Association for Computing Machinery, 21(3), 2002.

[15] T. Ju, S. Schaefer, J. Warren, "Mean Value Coordinates for closed triangular meshes", Journal ACM Trans. On Graphics (TOG), 24(3), pp.561-566, July 2005

[16] M. S. Floater, "Mean Value Coordinates", Comput., Aided Geom. Des, 20(1), pp.19-27

[17] Y. Lipman, D. Levin, D. Cohen-Or, "Green Coordinates", ACM Trans. Onm Graphics, 27(3), Article 78, pp.78:1-78:10, August 2008.\#

[18] A. Vukovic, P. Sewell, T. M Benson, "Generating radome geometries for full lightning protection studies", ESA Workshop on Aerospace EMC, 2019, Budapest. Available online https://ieeexplore.ieee.org/document/8788907

[19] P. Sewell, T. M. Benson, C. Christopoulos, D. W. P. Thomas, A Vukovic, J G. Wykes, "Transmission line modelling (TLM) based upon unstructured tetrahedral meshes", IEEE Trans. Microwave Theory and Tech. 53, pp.1919-1928, 2005.

[20] Jian-Ming Jin, “The Finite Element Method in Electromagnetics”, 2nd ed. New York: John Wiley \& Sons, 2002.

[21] A. Bossavit, "Yee-like schemes on a tetrahedral mesh, with diagonal lumping", Int. J. Numer. Modeling, vol. 12, no. 1-2, pp. 129-142, 1999.

[22] J. Keranen, J. Kangas, A. Ahola and L. Kettunen, "Implicit Yee-like scheme on tetrahedral Mesh", IEEE Trans. on Magnetics, vol. 38, no. 2, pp. 717-720, March 2002.

[23] J. Gao, G. Kobidze and B. Shanker, "Higher-order FDTD algorithm using tetrahedral Tessellation", Proc. IEEE AP-S Int. Antennas and Propagation Symp, Columbus, USA, vol. 4, , pp. 364 - 367, Jun 2003.

[24] P. Sewell, J. G. Wykes, T. M. Benson, C. Christopoulos, D. W. P. Thomas, A. Vukovic, "Multi-grid interface in computational electromagnetics", Electron. Lett, 40, pp. 162-163, 2004

[25] P. Sewell, A. Vukovic, X. Meng, T. M. Benson, "A note on material losses in Unstructured Transmission Line Modelling (UTLM)", Microwave and Optics Technology Letters, 57(9), pp.2218-2222, September 2015.

[26] X. Meng, A. Vukovic, T. M. Benson, P. Sewell, "Extended capability models for Carbon Fiber Composite (CFC) panels in the Unstructured Transmission Line Modelling (UTLM) Method", IEEE Transactions on Electromagnetic Compatibility 58 (3), 811-819, 2016.

[27] P. Sewell, A. Vukovic, T. M. Benson, X. Meng, "Complexity reduction of multiscale UTLM cell clusters", IEEE on Multiscale and Multiphysics Computational Techniques, 2, pp.18-28, February 2017.

[28] D. Jiao, A. A. Ergin, B. Shanker, E. Michielssen, J. -M. Jin, “A fast timedomain finite element-boundary integral method for electromagnetic analysis", IEEE Trans. Antennas Propagat., vol.49, no.10, pp.1453-1461, October 2001.

[29] P. Sewell, A. Vukovic, T. M. Benson, X. Meng, "Extracting Modal Field Profiles from 3D Unstructured TLM Meshes for use as Sources and
Observers", IET Science, Measurements \& Technology,

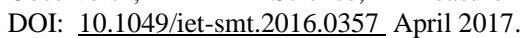

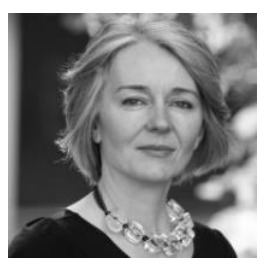

Ana Vukovic (M'97) was born in Nis, Serbia, in 1968. She received the Diploma of Engineering degree in electronics and telecommunications from the University of Nis, Nis, Yugoslavia, in 1992, and the Ph.D. degree from the University of Nottingham, Nottingham, U.K., in 2000. From 1992 to 2001, she was a Research Associate with the University of Nottingham. In 2001, she joined the School of Electrical and Electronic Engineering, University of Nottingham, as a Lecturer. In 2008 and 2020, she became an Associate Professor and Professor of Electromagnetic Applications at the University of Nottingham.Her research interests are electromagnetics with a particular emphasis on applications in optoelectronics, microwaves, EMC and aerospace.

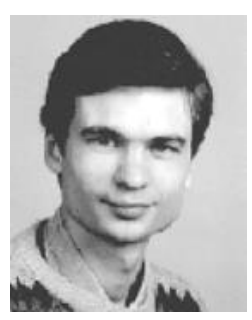

Phillip Sewell was born in London, U.K., in 1965. He received the B.Sc. degree in electrical and electronic engineering (with first-class honors) and Ph.D. degree from the University of Bath, Bath, U.K., in 1988 and 1991, respectively. From 1991 to 1993 , he was a Post-Doctoral Fellow with the University of Ancona, Ancona, Italy. In 1993, he became a Lecturer with the School of Electrical and Electronic Engineering, University of Nottingham, Nottingham, U.K. In 2001 and 2005, he became a Reader and Professor of electromagnetics at the University of Nottingham. His research interests involve analytical and numerical modeling of electromagnetic problems with application to optoelectronics, microwaves and aerospace applications.

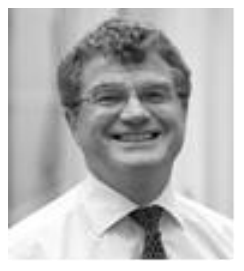

Trevor M. Benson received a First Class honors degree in Physics and the Clark Prize in Experimental Physics from the University of Sheffield in 1979, a PhD in Electronic and Electrical Engineering from the same University in 1982 and the DSc degree from the University of Nottingham in 2005.

After spending over six years as a Lecturer at University College Cardiff, Professor Benson moved to The University of Nottingham in 1989. He was promoted to a Chair in Optoelectronics in 1996, having previously been Senior Lecturer (1989) and Reader (1994). Professor Benson's research interests include experimental and numerical studies of electromagnetic fields and waves with particular emphasis on the theory, modeling and simulation of optical waveguides, lasers and amplifiers, nano-scale photonic circuits and electromagnetic compatibility. He is a Fellow of the Institute of Engineering Technology (FIET), the European Optical Society (FEOS) and the Institute of Physics (FInst.P). He was elected a Fellow of the Royal Academy of Engineering in 2005 for his achievements in the development of versatile design software used to analyze propagation in optoelectronic waveguides and photonic integrated circuits. 